\title{
The HeI $\lambda 10830 \AA$ Line as an Indicator of the Chromospheric and Coronal Activity of the Sun
}

\author{
A.G. Shcherbakov and Z.A. Shcherbakova \\ Crimean Astrophysical Observatory, \\ Nauchny, 334413, Crimea, USSR
}

\begin{abstract}
The behaviour of the HeI $\lambda 10830 \AA$ line profile in spectra of solar features is reviewed. The connection of line characteristics with various phenomena on the solar disc, such as plages (faculae), sunspots, jets, prominences, coronal holes, flares, fine structure and emission above the solar limb, is discussed. We present a short summary of the mechanisms for helium excitation in the solar chromosphere. Time variations of the line profile, observed in quiet and active regions, and HeI $\lambda 10830 \AA$ absorption in the Sun as a star are discussed and compared with other indexes of solar activity. It is shown that the equivalent width of the helium line varies in an 11 year cycle and reflects the 27 day periodicity of the rotation of the Sun. It is also shown that the HeI line is a more sensitive index of activity than $\mathrm{K}$-index.
\end{abstract}

\section{HeI $\lambda 10830 \AA$ line profile on the solar disc}

Observations of neutral helium lines in the spectrum of the Sun are limited by the high excitation potential of the atomic levels $(20 \mathrm{eV}$ or higher). These levels are populated rather poorly in the solar photosphere. Therefore the absorption occurs in chromospheric regions with low density and high temperature.

This is especially true for HeI $\lambda 10830 \AA$, a line which is formed in the chromosphere by metastable features of its lower level. A model of the helium atom, showing the basic transitions, is presented in Fig. 1. The HeI $\lambda 10830 \AA$ line is formed by the transition $2 s^{3} S_{1}-2 p^{3} P_{2,1,0}$ and is composed of three components with the ratio of $g f$ values $5: 3: 1$. The two primary components are separated only by $0.09 \AA$, thus being physically blended into one line at $\lambda 10830.3 \AA$. It is separated from its $\lambda 10829.1 \AA$ satellite by $1.26 \AA$. The effective ratio of $g f$-values for the two resolvable components is $8: 1$. Figure 2 shows a spectral region around the HeI $\lambda 10830$ line. 


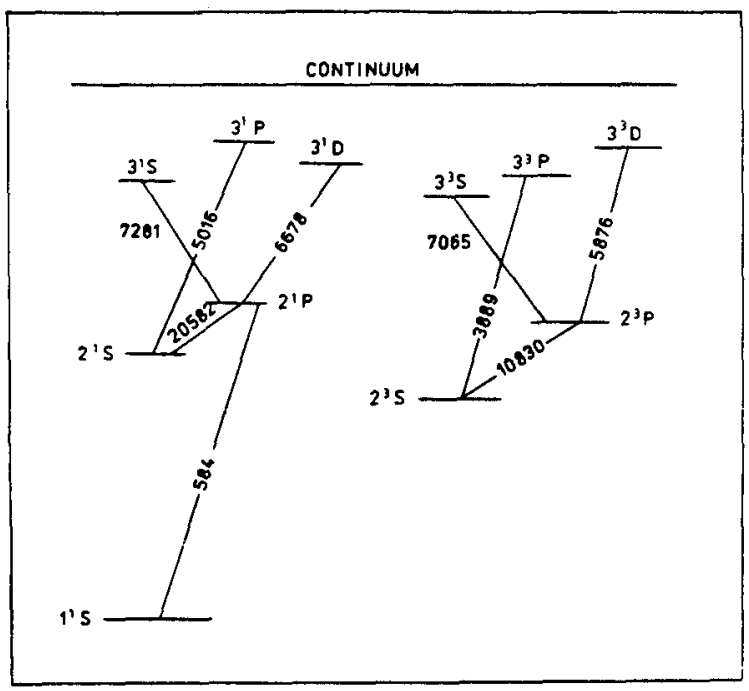

Fig. 1. Atomic levels of the HeI.

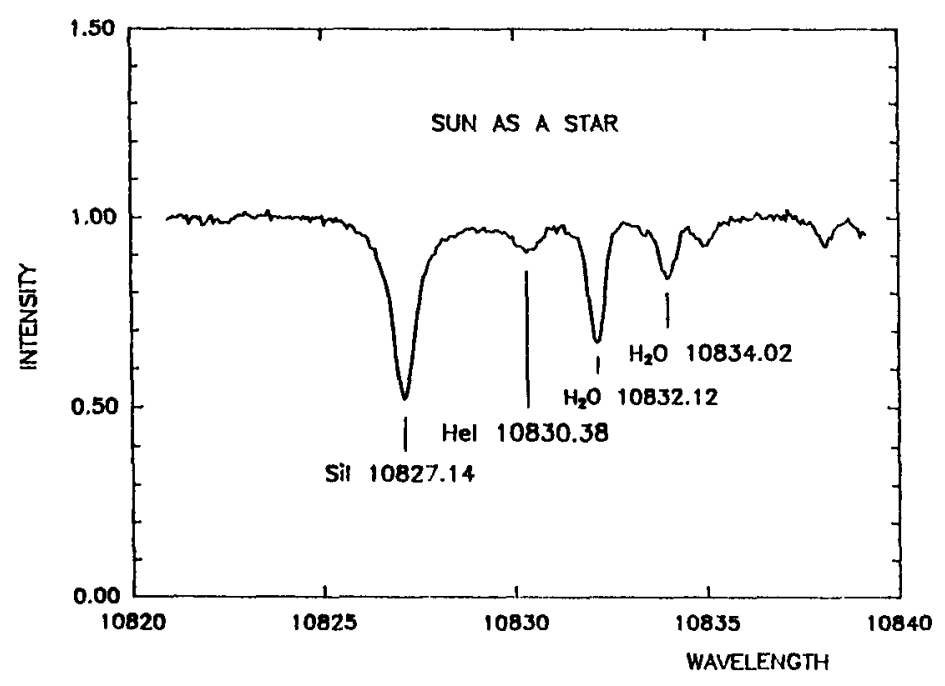

Fig. 2. The spectrum of the HeI $\lambda 10830 \AA$ absorption region.

Babcock and Babcock (1934) were the first to observe this line as a weak diffuse absorption in the spectrum of the Sun. That the line originates in strongly inhomogeneous regions with complicated fine structure such as dark faculae, filaments and traces of chromospheric network, was discovered by D'Azambuja and D'Azambuja (1938). 


\subsection{Variations over the disc and fine structure}

It was the equivalent width $W_{\lambda}$ of the line that was first studied in detail (Mohler and Goldberg, 1956). They found that the centre-to-limb variation of $W_{\lambda}$ in the equatorial direction was higher than in the polar direction. Delbouille et al. (1960) estimated $W_{\lambda}$ values for several points on the solar disc. Numerous measurements of $W_{\lambda}$ were carried out in 1961-1962 by Gulyaev (1964).

Detailed investigations of line profile in undisturbed regions by De Jager et al. (1966), showed that the ratio of component depths

$$
r=R_{0}^{\text {obs }}(10830) / R_{0}^{\text {obs }}(10829)
$$

over the whole disc is near to 4 (from 5 to 3 ) rather than the value $r=8$, which corresponds to optically thin gas. On the other hand, the depth of the line is very small ( $<10 \%$ in continuum units) implying a small number of absorbing atoms. This leads to a contradiction that can be solved assuming that $\mathrm{HeI}$ line is formed in unresolved structural inhomogeneities. In this case the observed line depth can be expressed as

$$
R_{0}^{\mathrm{obs}}(10830)=a R_{0}(10830),
$$

where the multiplier $a$ is the filling factor. These authors identified such an inhomogeneities with spicules. They suggest that the HeI line occurs in fine structures (spicule type) with equal optical depths and excitation temperature, and that the medium between spicules does not contribute into the formation of line. Their data yielded $a=0.12$. Nguen-Ngan (1970) came to the same conclusion. He postulated that the line is being formed partially in optically thin layers and partially in optically thick inhomogeneities $(\tau \sim 2)$, occupying about $5 \%$ of the disc. Giovanelli and Hall (1977) showed that the line depth is twice as large at the boundaries of supergranules than in their centres. According to their measurements the line depth at the boundaries of supergranules can reach $15 \%$.

Detailed analysis of line profile variations across the solar disc was carried out by Shcherbakova et al. (1983) and Shcherbakov and Shcherbakova (1983). Fig. 3 shows line profiles in the centre of the disc, at the limb and in a plage region, while Fig. 4 presents the variations of the depth and half width of the line in polar and equatorial directions. As shown in Fig. 4, in the north-south direction the line depth varies only slightly. The darkening on the limb becomes evident only at $\Theta>70^{\circ}$. From the comparison of variations of $R_{0}$ and $\Delta \lambda_{1 / 2}$, the main contribution to the equivalent width variations is due to the variation of half-width, but not depth (with the exception of highly disturbed active regions).

High resolution spectroheliograms by Rust and Bridgest (1975) showed even finer structure. The dark chromospheric network in HeI with an average cell size of 40 " coincides in general with the bright network in $\mathrm{H} \alpha$ and CaII, but even finer structure is displayed inside the chromospheric network. At the junctions of this network darker knots are observed that are identified with dark helium points (rather stable formations with the lifetime more than 24 hours), corresponding to bright X-ray points. Harvey and Sheeley (1977) found that the most intensified $\mathrm{He}$ regions lie at the bases of coronal magnetic loops. The appearance of He points in 

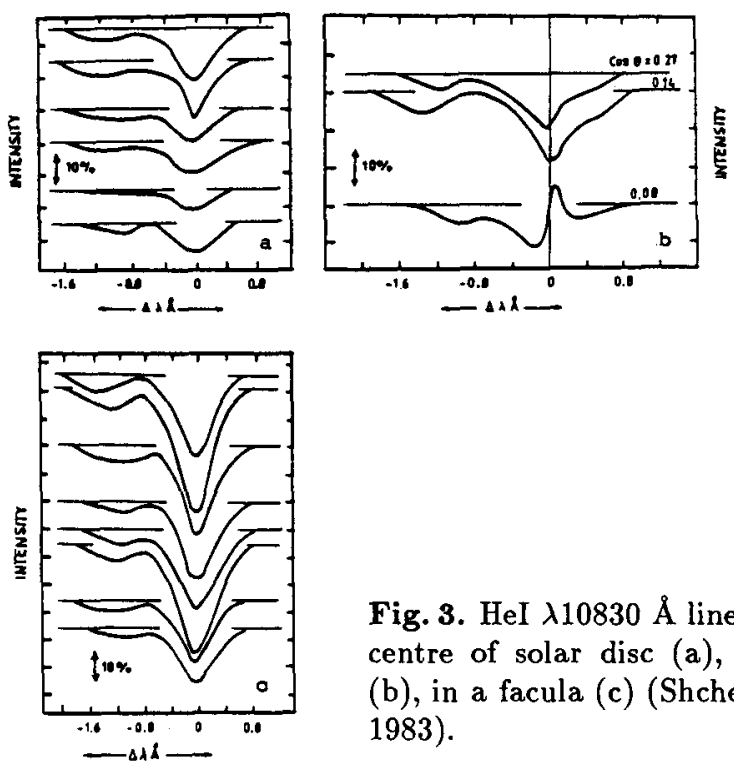

Fig. 3. HeI $\lambda 10830 \AA$ line profiles in the centre of solar disc (a), near the limb (b), in a facula (c) (Shcherbakova et al., 1983).

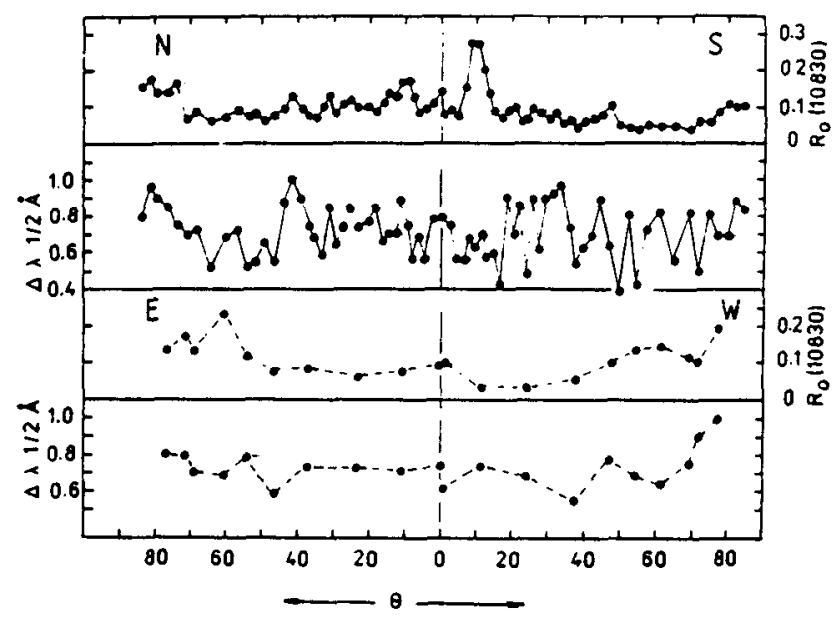

Fig. 4. Central depth and half-width variations of the HeI $\lambda 10830 \AA$ line on the solar disc (Shcherbakov and Shcherbakova, 1983).

the network nodes is caused by the presence of intensified magnetic field in these places. Harvey (1985) showed that about $1 / 3$ of the dark points correspond to sites of eruptive magnetic flux (ephemeral active regions), and the remainder to places where elements of the network of opposite polarities meet.

According to Rust and Bridgest (1975) absorption in the helium line has a correlation with the photospheric magnetic field configuration, though some differences are observed. Harvey and Hall (1971) also found a correlation between $\mathrm{He}$ 
line absorption and magnetic field intensity, with the greatest absorption being in places where there is a change of magnetic polarity.

\subsection{Plages (Faculae)}

In contrast to quiet regions, a strong absorption of the helium line is always observed in plages. Namba (1963) measured the line profiles in the spectra of 22 plage regions. He found that the average depth of the primary component of the line is $24 \%$ of the continuum intensity. Analogous results were obtained by Gulyaev (1964) and Nguen-Ngan (1970).

Shcherbakova et al. (1983) found that (Fig. 3) the depth and width of the line varies significantly inside plage regions. The mean values of $R_{0}(10830)$ for some plages does not exceed $30 \%$ while for others it exceeds 40 to $50 \%$. The evaluation of physical conditions in plage regions was carried out by assuming plage homogeneity and stability of excitation temperature. Nguen-Ngan computed the filling factor $a$ of absorption for plages as close to 0.5. According to Shcherbakova et al. (1983) the value of $a$ was 0.3 for two observed plages, and for two other plages it was near to unity.

\subsection{Sunspots}

Strong helium absorption ( $R=17 \%$ and 30\%) was found by Fay et al. (1972) in the chromospheric spectrum above two sunspots, the larger sunspot having greater line depth. They suggest that line intensification occurs due to chromospheric heating by magnetohydrodynamic waves, intensified in the spot umbra by its strong magnetic field. Borodina and Papushev (1979) also note the intensification of helium line over the centre of the spot $(R=20 \%)$.

At the Crimean Observatory we have investigated the line profile over the umbra and penumbra for ten sunspots (unpublished data). It was found that line strengths over the umbra of a spot and its depth changes from spot to spot, within the limits $16 \%-27 \%$. The ratio $r$ of the two components is, on average, about 4 which is the same as for plages.

On the other hand, Lites (1986) observed that the He line was an extremely weak line over a sunspot (7.5\%). After a correction for scattered light, Lites gave a value $R=10 \%$ and noted that this is close to the line depth in the undisturbed chromosphere.

\subsection{Emission above the limb}

The half-width of the absorption line $\lambda 10830 \AA$ increases toward the limb, with the centre of the line being filled by emission (Fig. 3) which changes to a full emission contour spreading wide above the limb.

According to Gulyaev (1964), the equivalent width of the line reaches a maximum value at $1400 \mathrm{~km}$, with the dependence on height above the plage being 
similar to that of quiescent regions. Shcherbakov and Shcherbakova (1983) obtained the same result $(1500 \mathrm{~km})$. According to Giovanelli and Hall (1977), line emission is maximum at a height of $1700 \mathrm{~km}$.

\subsection{Jets and prominences}

Besides plages, probably the only site on the solar disc where strong absorption in HeI $\lambda 10830 \AA$ is observed (up to $50 \%$ ) is in jets. Being a projection of intense eruptive prominences on the disc, jets display a complex asymmetrical absorption line and a broad velocity field. Individual jet knots are shifted in the line along the line of sight to $10-70 \mathrm{~km} / \mathrm{s}$ (Shcherbakova, unpublished data). Line half-width increases with the knot velocity along the line of sight, i.e. knots with higher velocities have higher internal motions (Fig. 5).

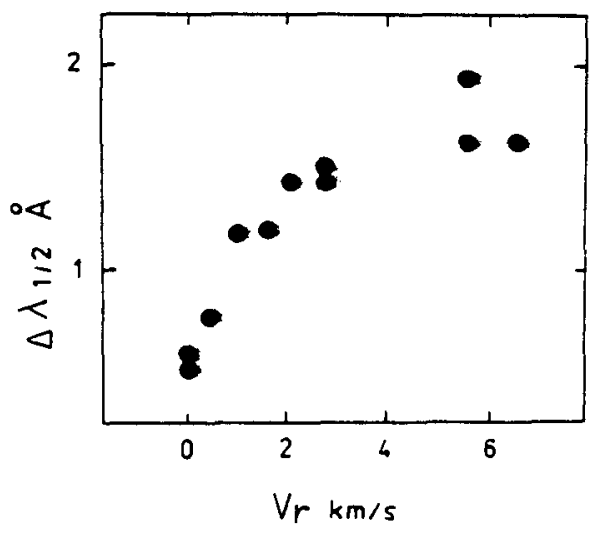

Fig. 5. The HeI $\lambda 10830 \AA$ line parameters in a dark jet on the solar disc.

Until now only quiet prominences have been investigated. They were observed by Tandberg-Hanssen (1962), Illing et al., (1975), Landman and Illing (1976), Landman (1976), Shcherbakova et al., (1983), Heinzel et al., (1986). It was found that the excitation temperature of the transition $2^{3} P-2^{3} S$ in prominences corresponds to $4020 \mathrm{~K}$ (according to Landman) and that optically thin wings in the line cannot be explained in terms of a simple isothermal model of prominences. A temperature increase in the transition layer between the prominence and corona must be accounted for (Heinzel et al., 1986).

\subsection{Flares}

Vaughan and Zirin (1968) showed that emission in the HeI $\lambda 10830 \AA$ line is possible only in dense regions $\left(n_{\mathrm{e}}>10^{12} \mathrm{~cm}^{-3}\right)$, where electron collisions become a significant source of quanta at $\lambda 10830$. In the solar chromosphere such conditions are realized only in regions connected with flares. Tandberg-Hanssen (1962) found 
that in weak flares the helium line is in absorption, and that only $2 \mathrm{~B}$ flares show emission.

Baranovsky and Shcherbakova (1985) investigated the emission line profile for $2 \mathrm{~B}$ and $2-3 \mathrm{~N}$ flares. Line emission for these flares reached $20 \%$ of the continuum. They showed that the formation region of $\mathrm{HeI} \lambda 10830, \mathrm{HeI} \lambda 10829$ and HeI $\lambda 584$ $\AA$ lines coincides with that of $\mathrm{L} \alpha-\mathrm{L} \gamma$ line formation and corresponds to a small optical depth $\tau \sim 0.25(r=7: 1)$

However, helium emission is not confined to powerful flares. Rust and Bridges (1975), while studying 12 subflares, found that above developing pores bright emission points (kernels) with a size of $3-5$ " appear in the HeI $\lambda 10830 \AA$ line. They came to the conclusion that dark disturbed fibrils in the $\mathrm{He}$ line, as well as emission points, coincide with fine structure of magnetic field. He flares develop in the regions about 5" across where there is an emerging magnetic field.

\subsection{Connection with coronal holes}

Solar images in the IR helium line look like a "negative copy" of the X-ray and far UV pictures. This similarity is most obvious for the negative image of the emission of the HeII $\lambda 304 \AA$ line (Harvey and Sheeley, 1977).

While identifying solar image in HeI $\lambda 10830$ line and soft X-rays, particularly during the SKYLAB mission, it became clear that by using the He line from ground-based observations, one can also observe coronal holes. Harvey et al. (1975) showed that the coronal holes in HeI $\lambda 10830 \AA$ line are displayed as regions with a "faded" or weakened chromospheric network.

According to Kahler et al. (1983) there is a correlation between hole boundaries in HeI $\lambda 10830$ line and X-rays, though they do not always coincide. Part of the boundaries may be highly diffuse. The best correspondence is observed for extended coronal holes while the correlation is rather poor for small holes in middle and low latitudes. The contrast of coronal holes in HeI line is not stable and depends upon the phase of solar activity.

\subsection{Time variations}

Sitnik et al. (1986) observed quiet and active regions on the solar disc from 1976 to 1985 . They found that in the quiet chromosphere the mean values of the helium line depth $R_{0}$ during maximum solar activity are $35-40 \%$ higher than during the period of increasing or decreasing activity. In the chromosphere above plages the values of $R_{0}$ increase by up to $15-20 \%$. Thus, during the solar maximum the "helium chromosphere" is intensified in quiet as well as in active regions on the Sun, and therefore the intensification of the HeI line in the spectrum of the Sun as a star is caused not only by the fraction of active chromosphere (number and dimension of active regions), but also by a general intensification of the helium chromospheric network. 


\section{HeI $\lambda 10830 \AA$ line variations in spectrum of the Sun as a star.}

In the mid 1960's techniques for observations of the "Sun as a star" were developed. For example, a variable magnetic field was detected on the Sun observed in this way. Rapid 27-day period fluctuations, connected with solar rotation, as well as long-term variations caused by solar activity, were discovered (Bumba et al., 1967). Magnetic field variability is explained mainly by the irregular distribution of largescale magnetic fields on the solar disc together with the evolution of background fields (Bumba and Howard, 1965).

Emission in the CaII $\mathrm{K}$ line for the whole solar disc is given by the $\mathrm{K}$-index. Wilson (1968) first defined the K-index and used it for the study of stellar cycles. The correlation between CaII and various active and quiet chromospheric structure was investigated and compared with a number of other solar indexes. For more detailed analysis of the problem one may see Bumba et al. (1967a), Sheeley (1967), Shcherbakova (1976), White and Livingston $(1978,1981)$ and Keil and Worden (1984).

A number of extensive long-term programmes for the study of solar activity indexes, from the far UV to IR regions, was initiated by the National Solar Observatory in the USA. Details of the results of these programmes appear in White (1988). For sunspot cycle 21 he computed indexes such as Wolf numbers, plagearea index, radio flux at $10.7 \mathrm{~cm}$, CaII K-index, $\mathrm{HeI} \lambda 10830$-index and UV fluxes in the regions $1000-4000 \AA$ and shortward of $1000 \AA$.

Figure 6 shows the comparison of data obtained by White and Livingston for the K-index (a) with the equivalent width of $\mathrm{HeI} \lambda 10830$ line measured approximately monthly by Livingston (b) the same except using daily values by Harvey (c). It is seen that the $\mathrm{K}$-index changes during the activity cycle by an average of $20 \%$. However, more rapid fluctuations, caused by passage of active regions across the solar disc, are also observed. According to Lean (1987) and White (1988), the amplitude of observed variability of $\mathrm{K}$-index cannot be explained entirely by the emission from chromospheric plages. Observed values of the K-index significantly exceed the total emission from all chromospheric plages. The spectrum of quiet regions at the centre of solar disc, according to White et al. (1987), shows only a slight variation during one cycle.

Thus, there must exist an additional source of CaII emission, contributing to the total emission of the Sun. Skumanich et al. (1984), Lean (1987) and White (1988) come to the conclusion that this additional source could be a so-called "active" chromospheric network appearing outside spots and plage fields. According to Leighton $(1964,1965)$ the "active" network consists of remnants from destroyed chromospheric plages and spots. These remnants, preserving their magnetic flux, contribute additional emission to the chromospheric network.

During the maximum of activity, the "helium chromosphere" is intensified not only in active regions on the solar disc, but also in quiet regions. Therefore the HeI $\lambda 10830$ line intensification in the spectrum of the Sun as a star is caused 
not only by the number and sizes of the active regions, but also by an additional contribution from the "active" chromospheric network. As it is seen from Fig. 6, variations of $\mathrm{HeI}$ line equivalent width have a good correlation with $\mathrm{K}$-index.
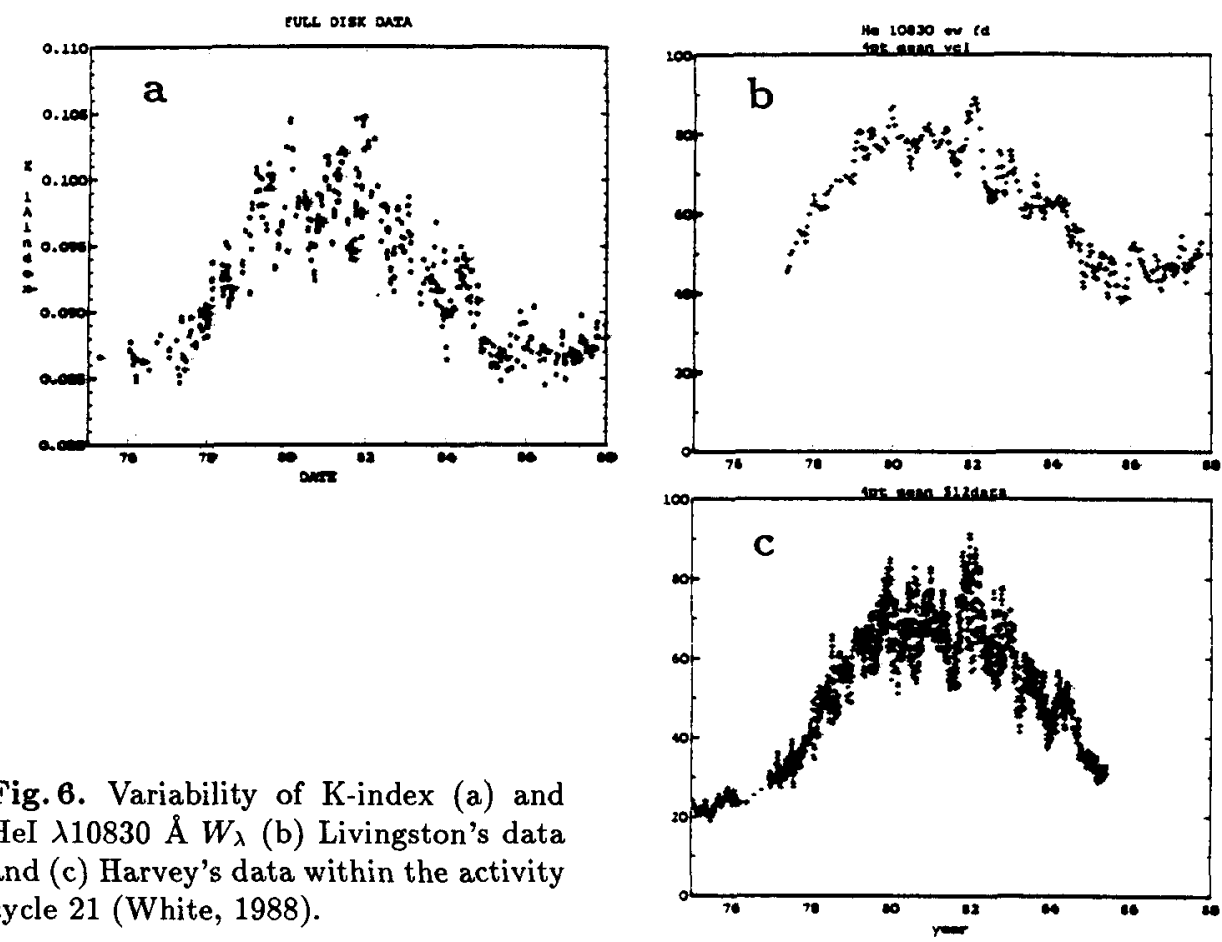

Fig. 6. Variability of $\mathrm{K}$-index (a) and HeI $\lambda 10830 \AA W_{\lambda}$ (b) Livingston's data and (c) Harvey's data within the activity cycle 21 (White, 1988).

It can see from Fig. 6 that the equivalent width of HeI line varies over the activity cycle by as much as a factor of 4 , whereas the $\mathrm{K}$-index shows a maximum amplitude of variability of only $29 \%$. Therefore it follows that the HeI line is a more sensitive index of activity than the K-index. White (1988) found a satisfactory correlation between both indexes and the UV flux from the Sun. Both indexes, in addition to variability connected with activity, show the 27-day period of rotation and correlate better with the UV flux than the radio flux at $10.7 \mathrm{~cm}$.

Similar results in the HeI $\lambda 10830 \AA$ line for the Sun as a star were obtained by Shcherbakova and Shcherbakov (1987), Shcherbakova et al. (1989) and Shcherbakov et al. (1990b) (see Fig. 7). According to their data the main parameter of the line, associated with solar activity, is line depth. For the period from 1981 to 1987 the line depth varied by more than a factor of 3 , whereas its half-width remained practically constant.

They confirm that the line depth shows day-to-day variations, with a dispersion that is twice as high in the period of maximum as in the period of minimum activity (see Fig. 7, also Figs. 6 b, c). This difference in amplitude is caused by the passage across the disk of inhomogeneous formations having different lifetimes and absorptions in the He-line. Close to the time of maximum activity the chromospheric network is disturbed by the birth and destruction of short-lived active 


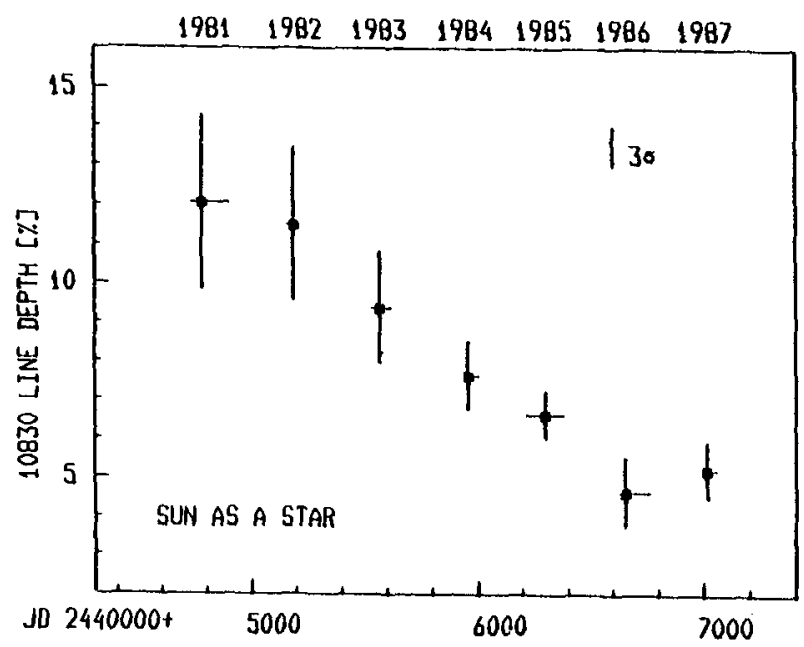

Fig. 7. Variability of the HeI $\lambda 10830 \AA$ line depth within the cycle 21 (Shcherbakov et al., 1990).

regions having strong absorption in the HeI line and intensified in the quiet chromosphere ("active network"). Near minimum, line depth is affected by the near zero absorption in long-lived coronal holes.

According to Shcherbakov et al. (1990) there exists a correlation between such indexes of activity as Wolf number, radio flux at $10.7 \mathrm{~cm}$ and FeXIV coronal index CI (Rybansky et al., 1988). The strongest correlation is observed between the line depth and coronal index $(r=0.87 \pm 0.03)$, and the weakest $(r=0.73 \pm 0.05)$ is with the Wolf number. There exists some correlation with the absolute value of the Sun's general magnetic field. The character of the correlation with the coronal index is shown in Fig. 8. As shown by Rybansky et al. (1988) the maximum of the coronal index does not coincide with the maximum of the Wolf number, being shifted by about 2 years. The positions of minima, however, coincide on both curves. This means that the times of increasing and decreasing of coronal index are closer to each other than the corresponding time of Wolf numbers where the time of increasing is always much shorter. This phase shift of the maximum relative to Wolf number is also observed for the HeI $\lambda 10830 \AA$ line (Harvey, 1984) and K CaII emission (Livingston and Wallace, 1987).

The latter circumstance is evidence in favour of a similarity of excitation mechanisms and heights of formation (upper chromosphere, transition region chromosphere-corona) for the indexes in question. The coronal index Fe XIV $\lambda 5303$ $\AA$ is determined by the forbidden transition ${ }^{2} P_{3 / 2}-{ }^{2} P_{1 / 2}$ and reflects the activity of the lower coronal layers. On the other hand, the absorption in the HeI $\lambda 10830$ line, which is formed by to transition $2_{\mathrm{s}}^{2} S_{1}-2_{\mathrm{p}}^{3} P_{2,1,0}^{0}$ is controlled by radiation shorter than $\lambda 504 \AA$. The metastable level $2^{3} S$ has a high excitation potential and needs electron temperatures of the order of 10000-20000 K. Line weakening 


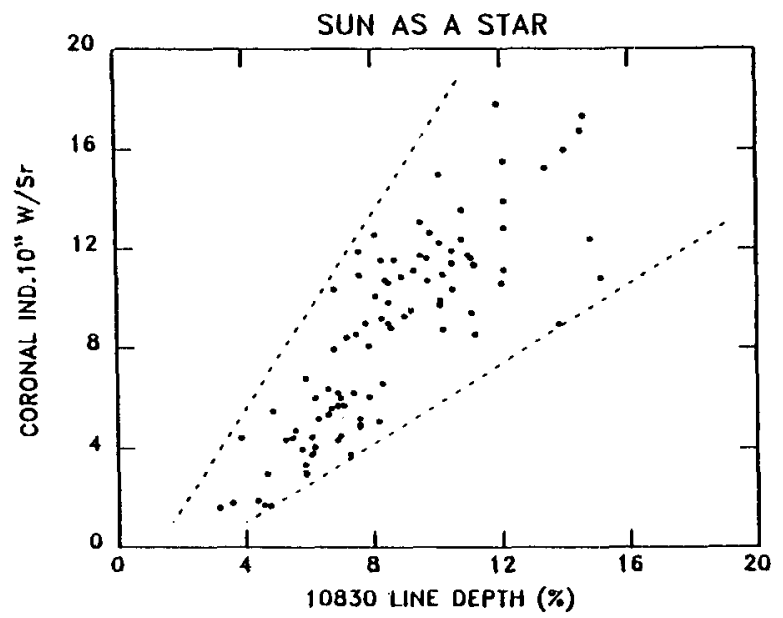

Fig. 8. The correlation between HeI $\lambda 10830 \AA$ line depth and Fe XIV coronal index within the cycle 21 (Shcherbakov et al., 1990).

is associated with the decrease of coronal UV back radiation from the corona onto the chromosphere in regions of coronal holes (Zirin, 1975, Livshits, 1975).

\section{Helium excitation}

Energy levels for the HeI atom are given in Fig. 1. Optical intercombination transitions between singlet and triplet levels in helium are forbidden, therefore the level $2^{3} S$ is highly metastable. The level $2^{1} S$ is also metastable, but in the conditions of the solar chromosphere it has an optical connection with the primary level through the $2^{1} P$ level ; the transition $2^{1} S-2^{1} P$ is realized while absorbing a quantum of $\lambda 20582$ Aphotospheric radiation while the transition $2^{1} P-1^{1} S$ appears spontaneously. Because of the absence of an optical connection, the triplet and singlet systems are practically independent. As a result, helium turns out to be composed of two sorts of atoms: parahelium (IP $=24.6 \mathrm{eV}$ ) and orthohelium $(\mathrm{IP}=4.7 \mathrm{eV}$ ), and their corresponding triplet levels appear to be overpopulated compared to singlets.

Weakening of the chromospheric HeI network in regions of coronal holes points to the connection with ionization by UV flux. The problem of the existence of helium ionization by short-wave radiation was considered firstly by Goldberg (1939) and then by Shklovsky (1951). After it became clear that triplet absorption lines appear due to the scattering of photospheric radiation (Krat and Sobolev (1960), Johnson (1960)), several authors (see Nickolskaya (1966) for spicules) developed the following mechanism of helium excitation: the neutral atom is ionized by emission with $\lambda<504 \AA$ followed by the recombination and delay for atoms in the metastable level $2^{3} S$.

On the other hand, knowing more about the nature of transition layers, it became possible to explain the appearance of regions with $T>2 \cdot 10^{4} \mathrm{~K}$ at low 
altitudes in the chromosphere, where the excitation and ionization of helium are caused by electron collisions (Athay and Johnson, 1960, Milkey et al., 1973). Milkey et al. propose two mechanisms: about half of the opacity in the absorption line appears in high temperature regions, where collisions populate the $2^{3} S$ level, and the other half occurs in low-temperature regions, where this level is populated by recombinations.

Gulyaev (1968) computed the concentration of HeI, HeII and HeIII atoms and the population of low levels of orthohelium for two extreme conditions: 1) the ionized emission from the corona back onto the chromosphere is absent; 2) the hard coronal emission is present. The optimal interval for orthohelium excitation appeared to be in the temperature range $20000-50000 \mathrm{~K}$. On the other hand, the observed orthohelium concentration might be generated also in cool regions $(T<10000 \mathrm{~K})$ by the photoionization of $\mathrm{HeI}$ followed by a recombination into triplet levels. Later Gulyaev (1972), from the analysis of Lyman distribution in HeI $(\lambda<504 \AA)$, determined that the electron temperature in quiet helium regions is equal to $12500 \mathrm{~K}$. Such a low value of $T_{e}$ is a serious argument for the role of UV coronal emission in leading to helium ionization. Livshitz (1975), from the analysis of the population of the $2^{3} S$ level in flocculi, also showed the advantage of the coronal UV emission excitation mechanism of the metastable level.

On the basis of a non-LTE computation of HeI populations, and using the 10830 line depth, Pozhalova (1987) demonstrated the possibility of making a quantitative estimation of the flux of coronal emission back onto the chromosphere. The VALC (Vernazza et al.,1982) model atmosphere was used with additional coronal UV illumination flux considered as a parameter. Computations showed that the UV flux really affects the population of the HeI atom. Therefore it is reasonable to expect that the weakening of UV flux in the region of coronal holes would lead to the weakening of $\mathrm{He} \lambda 10830$ line there.

Using the above ideas Pozhalova was the first to calculate HeI line profiles in the quiet chromosphere for various values of background coronal radiation. According to her computations the helium line in quiet regions on the Sun is optically thin.

\section{Conclusion}

We mention that the study of spectrum of the Sun as a star can be helpful for solving a number of problems of chromospheric and coronal activity of late type stars. Investigations of time variability of the IR HeI line profile in the spectra of different surface structures of the Sun, together with HeI observations in spectrum of the Sun as a star, might lead to the integral flux calibration for the Sun itself and for other stars (solar type stars in particular). Investigations of a number of $\mathrm{G}-\mathrm{K}$ and some $\mathrm{M}$ stars show that the helium line is observed in absorption as well as in emission. There is strong evidence of its variability with time (Vaughan and Zirin, 1968; Zirin, 1976; Zirin, 1982; Shcherbakov, 1979; Katsova and Shcherbakov, 1983; Shcherbakov et al., 1990a). 


\section{References}

Athay R.G., Johnson H.R.: 1960, Astrophys. J. 131, 413

Babcock H.D., Babcock H.W.: 1934, Publ. Astron. Soc. Pacific 46, 132

Baranovsky E.A., Shcherbakova Z.A.: 1985, Izv. Krym. Astrofiz. Obs. 71, 54 (Bull. Crimean Astrophys. Obs. 71, 52)

Borodina O.A., Papushev R.Zh.: 1979, Pis'ma Astron. Zh. 5, 620 (Soviet Astron. Letters 5,332 )

Bumba V., Howard R.: 1965, Astrophys. J. 141, 1502

Bumba V., Howard R., Smith S.F.: 1967, in "The Sun as a Magnetic Star", ed. C. Cameron, Mono Book, Baltimore, p. 131

Bumba V., Ruzickova-Topolova V.: 1967, Solar Phys. 1, 216

D'Azambuja L., D'Azambuja M.: 1938, Bull. Astronomique 11, 349

De Jager C., Namba O., Neven L.: 1966, Bull. Astron. Inst. Netherl. 18, 128

Delbouille L., de Jager C., Neven L.: 1960, Ann. d'Astrophys. 23, 949

Fay T.D., Willer A.A., Yun H.S.: 1972, Solar Phys. 23, 58

Giovanelli R.G., Hall D.: 1977, Solar Phys. 52, 211

Goldberg L.: 1939, Astrophys. J. 89, 673

Gulyaev R.A.: 1964, Astron. Zh. 41, 313 (Soviet Astron. 8, 243)

Gulyaev R.A.: 1968, in Solar activity, eds. E.R. Mustel, E.I. Mogilevski, I.A. Zulin, Nauka, Moscow, p. 104

Gulyaev R.A.: 1972, Solar Phys. 24, 72

Harvey K.L.: 1985, Austr. J. Phys. 38, 875

Harvey J.W.: 1984, in "Solar Irradiance Variations on Active Region Time Scales", NASA Conf., eds. B.J. Labonte, G.A. Chapman, H.S. Hudson, R.C. Wilson, p. 197

Harvey J., Hall D.: 1971, in "Solar Magnetic Fields", IAU Symp. 43, ed. R. Howard, Reidel, Dordrecht, Holland, p. 279

Harvey J.W., Sheeley N.R.: 1977, Solar Phys. 54, 343

Harvey J.W., Krieger A.S., Davis J.M., Timothy A.F., Vaiana G.S.: 1975, Bull. American Astron. Soc. 7, 358

Heinzel P., Kotrch P., Sobotka M., Zloch F., Shcherbakova Z.A.: 1986, Contrib. Astron. Obs. Skalnate Pleso 15, 171

Illing R.M.E., Landman D.A., Mickey D.L.: 1975, Solar Phys. 45, 339

Johnson H.R.: 1960, Ann. d'Astrophys. 23, 838

Kahler S.W., Davis J.M., Harvey J.W.: 1983, Solar Phys. 87, 47

Katsova M.M., Shcherbakov A.G.: 1983, Astron. Zh. 60, 267 (Soviet Astron. 27, 153)

Keil S.L., Worden S.P.: 1984, Astrophys. J. 136, 211

Krat V.A., Sobolev V.M.: 1960, Izv. Astron. Obs. Pulkovo 21, 2

Landman D.A.: 1976, Solar Phys. 50, 383

Landman D.A., Illing R.M.E.: 1976, Astron. Astrophys. 49, 277

Lean J.L.: 1987, Journ. Geophys. Res. 81, D1, 839

Leighton R.B.: 1964, Astrophys. J. 140, 1547

Leighton R.B.: 1965, in "Stellar and Solar Magnetic Fields", IAU Symp. No 22, ed. R. Lüst p. 158

Lites B.W.: 1986, Astrophys. J. 301, 1005

Livingston W.C., Wallace L.: 1987, Astrophys. J. 314, 808

Livshits M.A.: 1975, Astron. Zh. 52, 970 (Soviet Astron. 19, 587)

Milkey R.W., Heasley J.N., Beebe H.A.: 1973, Astrophys. J. 186, 1043

Mohler O.C., Goldberg L.: 1956, Astrophys. J. 124, 13 
Namba O.: 1963, Bull. Astron. Inst. Netherl. 17, 93

Nguen-Ngan: 1970, Astron. Zh. 47, 351 (Soviet Astron. 14, 283)

Nickolskaya K.I.: 1966, Astron. Zh. 43, 936 (Soviet Astron. 10, 751)

Pozhalova Zh.A.: 1987, Pis'ma Astron. Zh. 13, 610 (Soviet Astron. Letters 13, 255)

Rust D.M., Bridges C.A.: 1975, Solar Phys. 43, 129

Rybansky M., Rusin V., Dzifcakova E.: 1988, Bull. Astron. Inst. Czechosl. 39, 106

Shcherbakov A.G.: 1979, Pis'ma Astron. Zh. 5, 542 (Soviet Astron. Letters 5, 290)

Shcherbakov A.G., Shcherbakova Z.A.: 1983, Izv. Krym. Astrofiz. Obs. 68, 39 (Bull. Crimean Astrophys. Obs. 68, 37)

Shcherbakov A.G., Tuominen I., Jetsu L., Katsova M.M., Poutanen M.: 1990a, Astron. Astrophys. 235, 205

Shcherbakov A.G., Shcherbakova Z.A., Tuominen I., Rybansky M., Rusin V.: 1990b, Astron Astrophys. (to be submitted)

Shcherbakova Z.A.: 1976, Izv. Krym. Astrofiz. Obs. 55, 37

Shcherbakova Z.A., Shcherbakov A.G.: 1987, Izv. Krym. Astrofiz. Obs. 76, 98 (Bull. Crimean Astrophys. Obs. 76, 110 )

Shcherbakova Z.A., Shcherbakov A.G., Shapiro B.I., Heinman A.S.: 1983, Izv. Krym. Astrofiz. Obs. 66, 119 (Bull. Crimean Astrophys. Obs. 66, 109)

Sheeley N.R.: 1967, Astrophys. J. 147, 1106

Shklovsky I.S.: 1951, Ann. Sternberg Astron. Inst. 20, 5

Sitnik G.F., Kozlova L.M., Divlikeev M.I., Porfiryeva G.A.: 1986, Solnechnie Dannie, No 12,67

Skumanich A., Lean J.L., White O.R., Livingston W.C.: 1984, Astrophys. J. 282, 776

Tanberg-Hanssen E.: 1962, Ann. d'Astrophys. 25, 357

Vaughan A.H., Zirin H.: 1968, Astrophys. J. 152, 123

Vernazza J.E., Avrett E.H., Loeser R.: 1982, Astrophys. J. Suppl. 45, 635

White O.R.: 1988, in "Solar Radiative Output Variations", ed. P. Foukal, Cambridge Res. and Instr. p. 88

White O.R., Livingston W.C.: 1978, Astrophys. J. 226, 679

White O.R., Livingston W.C.: 1981, Astrophys. J. 249, 798

White O.R., Livingston W.C., Wallace L.: 1987, J. Geophys. Res. 92, D1, 823

Wilson O.C.: 1968, Astrophys. J. 153, 221

Zirin H.: 1975, Astrophys. J. 199, 63

Zirin B.: 1976, Astrophys. J. 208, 414

Zirin H.: 1982, Astrophys. J. 260, 655 